Pacific

Journal of

Mathematics

THE VOLUME-PRESERVING MEAN CURVATURE FLOW IN EUCLIDEAN SPACE

HAOZHAO LI

Volume $243 \quad$ No. 2

December 2009 


\title{
THE VOLUME-PRESERVING MEAN CURVATURE FLOW IN EUCLIDEAN SPACE
}

\author{
HAOZHAO LI
}

\begin{abstract}
We study the convergence of the volume-preserving mean curvature flow of hypersurfaces in Euclidean space under some initial integral pinching conditions. We prove that if the traceless second fundamental form is sufficiently small, the flow will exist for all time and converge exponentially fast to a round sphere.
\end{abstract}

\section{Introduction}

This paper proves the convergence of the volume-preserving mean curvature flow of hypersurfaces under some initial integral pinching conditions. Throughout, we discuss only closed, orientable, immersed hypersurfaces in Euclidean space.

Let $M$ be a compact $n$-dimensional manifold smoothly immersed in $\mathbb{R}^{n+1}$, and suppose $M$ is represented locally by an immersion

$$
F_{0}: \mathbb{R}^{n} \supset U \rightarrow F_{0}(U) \subset \mathbb{R}^{n+1} .
$$

We evolve the family of maps $F_{t}(x)$ by the equation and initial condition

$$
\partial_{t} F_{t}(x)=(h-H) v(x, t) \quad \text { and }\left.\quad F_{t}(x)\right|_{t=0}=F_{0}(x),
$$

where $v(x, t)$ is the outer unit normal vector on $M_{t}=F_{t}(M), H(x, t)$ is the mean curvature, and $h(t)$ is its average defined by

$$
h(t)=\frac{1}{V_{t}} \int_{M_{t}} H(t) d V_{t} .
$$

Here $V_{t}$ denotes the volume of $M_{t}$ with respect to the induced metric $g$. We denote by $A=\left\{h_{i j}\right\}$ the second fundamental form of $M$. We denote its traceless part $A-(H / n) g$ by $\AA$, and we usually use the norm $|\AA|^{2}=|A|^{2}-(1 / n) H^{2}$. For more detailed definitions, see Section 2.

For the volume-preserving mean curvature flow or general mean curvature flow, most of the work has focused on convexity conditions. G. Huisken [1987] (and Gage [1986] for curves) proved that if the initial hypersurface is compact and

Keywords: volume-preserving mean curvature flow, stability. 
uniformly convex, the flow will converge exponentially fast to a round sphere. In an asymptotically flat manifold with positive mass, Huisken and Yau [1996] proved convergence of the flow for an initial coordinate sphere with sufficiently large radius, and Rigger [2004] proved analogous results in the asymptotically hyperbolic setting. For nonconvex cases, Escher and Simonett [1998] proved that if the initial hypersurface $M_{0}$ is sufficiently close to a fixed Euclidean sphere, the flow will converge exponentially fast to a round sphere, and Cabezas-Rivas and Miquel [2007] discussed analogous results in hyperbolic space. For a general ambient manifold, Alikakos and Freire [2003] proved long time existence and convergence to a constant mean curvature surface under the hypotheses that the initial hypersurface is close to a small geodesic sphere and that it satisfies some nondegeneracy conditions.

The proofs in [Escher and Simonett 1998; Alikakos and Freire 2003; CabezasRivas and Miquel 2007] are based on center manifold analysis, and therefore leave unclear how the shape of the initial hypersurface affects the convergence of the flow. It would be preferable - and interesting - to have proofs of convergence that start with natural conditions on the geometry of the initial hypersurface. In this paper, we borrow the ideas from Ricci flow [Min-Oo 1990; Ye 1993] and Kähler-Ricci flow [Chen 2006; Chen et al. 2009; Chen and Li 2009] to prove convergence of the flow under conditions on the initial second fundamental form. Here is our main result:

Theorem 1. Let $M \subset \mathbb{R}^{n+1}$ be a compact, orientable, smoothly immersed hypersurface of dimension $n \geq 2$ satisfying

$$
V \leq v, \quad|A| \leq \Lambda, \quad h \geq \gamma, \quad \int_{M}|\AA|^{2} \leq \epsilon
$$

for any positive constants $\Lambda, \gamma$ and $v$ and sufficiently small $\epsilon=\epsilon(\Lambda, \gamma, v)>0$, where $V$ is the volume of $M$ with respect to the induced metric. Then the volumepreserving mean curvature flow (1-1) with the initial hypersurface $M$ will converge exponentially fast to a round sphere.

Remark 2. Using center manifold analysis, Escher and Simonett [1998] proved analogous results under a somewhat different condition, that $M$ is "sufficiently close" to a standard sphere in $h^{1+\beta}$ norm.

Remark 3. Unlike the results of Ricci flow in [Ye 1993; Chen and Li 2009] or mean curvature flow in [Huisken and Yau 1996; Rigger 2004], we don't need to assume any stability conditions of the initial hypersurface. In Theorem 1, the assumption on $h$ seems quite natural since $h$ is always positive if the initial hypersurface is close to a sphere. 
Turning to the results of Huisken and Yau [1996] for large coordinate spheres in an asymptotically Euclidean manifold and of Alikakos and Freire [2003] for small geodesic balls in a general Riemannian manifold, we find that our method can be used in these two cases with rather weak conditions. Here, we only give the result in Euclidean space, which is a direct corollary of Theorem 1:

Corollary 4. (1) For any $B_{1}, B_{2}, B_{3}, B_{4}$ and $\delta, \sigma>0$, we define the set of compact, orientable, smoothly immersed hypersurfaces

$$
B_{\sigma}=\left\{M^{n} \subset \mathbb{R}^{n+1}|||x|-\sigma\left|\leq B_{1},\right| \AA\left|(x) \leq \frac{B_{2}}{\sigma^{1+\delta}},\right| \nabla \AA \mid \leq \frac{B_{3}}{\sigma^{2+\delta}}, V \leq B_{4} \sigma^{n}\right\} .
$$

Then there is a constant $\sigma_{0}=\sigma_{0}\left(B_{1}, B_{2}, B_{3}, B_{4}, \delta\right)>0$ such that for all $\sigma \geq \sigma_{0}$, the volume-preserving mean curvature flow with any initial hypersurface in $B_{\sigma}$ will converge exponentially fast to a round sphere.

(2) For any $B_{1}, B_{2}, B_{3}, B_{4}$ and $\delta, \sigma>0$, we define the set of compact orientable, smoothly immersed hypersurfaces

$B_{\sigma}^{\prime}=\left\{M^{n} \subset \mathbb{R}^{n+1}|||x|-\sigma\left|\leq B_{1} \sigma^{1+\delta},\right| \AA\left|(x) \leq \frac{B_{2}}{\sigma^{1-\delta}},\right| \nabla \AA \mid \leq \frac{B_{3}}{\sigma^{2-\delta}}, V \leq B_{4} \sigma^{n}\right\}$.

Then there is a constant $\sigma_{0}=\sigma_{0}\left(B_{1}, B_{2}, B_{3}, B_{4}, \delta\right)>0$ such that for all $\sigma \in\left(0, \sigma_{0}\right)$, the volume-preserving mean curvature flow with any initial hypersurface in $B_{\sigma}^{\prime}$ will converge exponentially fast to a round sphere.

Huisken and Yau [1996] proved the convergence of the flow (1-1) under the assumption that the mass is positive, which implies the initial coordinate sphere is strictly stable. This assumption is crucial in their proof since it leads to the exponential decay of the $L^{2}$ norm of $|H-h|$. In the proof of Corollary 4 and Theorem 1, we avoid this by proving first the exponentially decay of the traceless second fundamental form for short time.

The proof of Theorem 1 uses methods similar to those in [Ye 1993; Chen et al. 2009; Chen and Li 2009]. First, we prove that under conditions (1-2), both $|\AA|$ and $|H-h|$ are small after a short time (see Proposition 13) and therefore decay exponentially in a short time interval provided that the average of the mean curvature is strictly positive (see Lemmas 15 and 16). On the other hand, that they decay exponentially implies that they are both small in the next time interval, and we can repeat the previous arguments. Using this iteration idea, we can prove both long time existence and exponential decay for all time.

Next we would like to weaken the initial condition on $|A|$. To remove it would be too much - in the general situation without an initial bound, one cannot even expect uniform short time existence of the flow. We overcome this difficulty using the ideas of $\epsilon$-regularity of mean curvature flow in [Ecker 1995; Nakauchi 1993] and Willmore flow in [Kuwert and Schätzle 2002], and we replace the bound on $|A|$ 
by a bound on mean curvature or its average. We can then prove that the flow has uniform short time existence and that the second fundamental form is bounded after a short time. For simplicity of the proof, we only state the result when $n=2$ :

Theorem 5. Let $M^{2} \subset \mathbb{R}^{3}$ be a compact, smoothly immersed surface with

$$
\text { (1-3) } 0<v_{0}<V<v_{1}, \quad 0<\gamma<h<\Lambda, \quad \int_{M}|H-h|^{2} \leq \epsilon, \quad \int_{M}|\AA|^{2} \leq \epsilon
$$

for any positive constants $v_{0}, v_{1}, \Lambda, \gamma$ with $v_{0}<v_{1}, \gamma<\Lambda$, and sufficiently small $\epsilon=\epsilon\left(\gamma, \Lambda, v_{0}, v_{1}\right)>0$. Then the volume-preserving mean curvature flow with initial surface $M$ will converge exponentially fast to a round sphere.

Remark 6. The assumption (1-3) can be replaced by

$$
v_{0}<V<v_{1}, \quad \gamma<H<\Lambda, \quad \int_{M}|\AA|^{2} \leq \epsilon .
$$

The proof is similar to that of Theorem 5 but is rather long, so we omit it.

Remark 7. For higher-dimensional cases, the idea is the same, but we may assume the $L^{n}$ norms of $|\AA|$ and $|H-h|$ are small; see [Nakauchi 1993]. The idea can also be applied to the normalized Ricci flow (see [Ye 1993]), where we can replace the bound on the full Riemann curvature tensors by the smallness of the $L^{n / 2}$ norms of Weyl tensors and the traceless Ricci curvature.

Roughly, the proof of Theorem 5 is as follows: We hope to use the $\epsilon$-regularity to prove that the conditions (1-2) in Theorem 1 are satisfied after a short time. First, we prove that the $L^{p}$ norms of $|\AA|$ and $|H-h|$ are small for a short time interval under the conditions (1-3). Then standard $\epsilon$-regularity together with Michael and Simon's Sobolev inequality implies that the $L^{\infty}$ norm of $|A|$ is bounded after a short time, and we can apply Theorem 1 to prove the convergence.

In forthcoming papers, we expect to generalize this method to hypersurfaces in asymptotically flat manifolds and asymptotically hyperbolic manifolds without necessarily positive mass. Recently, Mazzeo and Pacard [2007] proved very interesting results for the construction of constant mean curvature foliations on conformally compact manifolds, and we expect that our method works in this setting. Our method can also be used for Lagrangian mean curvature flow and symplectic mean curvature flow, and we will explore this in the future.

\section{Notation and preliminary results}

We follow the notation of [Huisken 1984]. Define the manifold $M$ and the family of maps $F_{t}$ as on page 331 . Since $M_{t}$ is given locally by $F$, we can calculate

$$
g_{i j}(x)=\left(\frac{\partial F}{\partial x^{i}}, \frac{\partial F}{\partial x^{j}}\right) \quad \text { and } \quad h_{i j}=-\left(v(x, t), \frac{\partial^{2} F}{\partial x^{i} \partial x^{j}}\right) \quad \text { for } x \in \mathbb{R}^{n} .
$$


Using these notations, the mean curvature and the norm of second fundamental form are given by $H=g^{i j} h_{i j}$ and $|A|^{2}=g^{i j} g^{k l} h_{i k} h_{j l}$.

We now collect some well-known identities. The induced metric evolves by

$$
\partial_{t} g_{i j}=2(h-H) h_{i j}
$$

Hence, the volume form of $M_{t}$ satisfies

$$
\frac{\partial}{\partial t} d V_{t}=H(h-H) d V_{t} \text {. }
$$

Applying the Codazzi equations and interchanging derivatives, we can prove these identities:

Lemma 8. $\partial_{t} H=\Delta_{t} H+(H-h)|A|^{2}$,

$$
\begin{aligned}
\partial_{t} h_{i j} & =\Delta_{t} h_{i j}-2 H h_{i k} h_{k j}+h h_{i k} h_{k j}+|A|^{2} h_{i j}, \\
\partial_{t}|A|^{2} & =\Delta|A|^{2}-2|\nabla A|^{2}+2|A|^{4}-2 h \operatorname{tr}\left(A^{3}\right) .
\end{aligned}
$$

Lemma 9. $\partial_{t}|\AA|^{2}=\Delta|\AA|^{2}-2|\nabla \AA|^{2}+2|A|^{2}|\AA|^{2}-2 h\left(\operatorname{tr}\left(\AA^{3}\right)+\frac{2}{n} H|\AA|^{2}\right)$,

$$
\partial_{t}|\nabla \AA|^{2}=\Delta|\nabla \AA|^{2}-2\left|\nabla^{2} \AA\right|^{2}+\nabla A * \nabla \AA * A * \AA+\nabla \AA * \nabla \AA * A * A,
$$

where $A * B$ denotes the contraction of the tensors $A$ and $B$.

Proof. By Lemma 8 a straightforward computation implies

$$
\partial_{t}|\AA|^{2}=\Delta|\AA|^{2}-2|\nabla \AA|^{2}+2|A|^{2}|\AA|^{2}-2 h\left(\operatorname{tr}\left(A^{3}\right)-(1 / n)|A|^{2} H\right) .
$$

Observe that in a coordinate system where $h_{i j}=\kappa_{i} g_{i j}$, the eigenvalues of the traceless second fundamental form are given by

$$
\mu_{i}=\kappa_{i}-H / n \quad \text { and } \quad \sum_{i} \mu_{i}=0 .
$$

Then the first identity follows from (2-3) and

$$
\begin{aligned}
\operatorname{tr}\left(A^{3}\right)-(1 / n)|A|^{2} H & =\sum_{i}\left(\mu_{i}+\frac{H}{n}\right)^{3}-\frac{H}{n}\left(\mu_{i}+\frac{H}{n}\right)^{2}=\sum_{i}\left(\mu_{i}^{3}+\frac{2}{n} H \mu_{i}^{2}\right) \\
& =\operatorname{tr}\left(\AA^{3}\right)+(2 / n) H|\AA|^{2} .
\end{aligned}
$$

The second identity follows from [Huisken 1984].

\section{Proof of Theorem 1 and Corollary 4}

The second fundamental form. Here, we prove that under assumption (1-2), the pointwise bound on the traceless second fundamental form will be small after a short time. We need to use the parabolic Moser iteration for the mean curvature flow in the form found in [Dai et al. 1996; Ye 1993; Chen and Tian 2006]: 
Theorem 10. Let $f$ be a nonnegative function satisfying

$$
\partial_{t} f \leq \Delta f+b f \quad \text { for } t \in[0, T],
$$

where $b$ is a nonnegative constant and $\Delta$ is the Laplacian of the induced metric $g(t)$ of the mean curvature flow (1-1). Then

$$
f(t) \leq C(n, \Lambda, b, \tau)\left(\int_{t-\tau}^{t} \int_{M} f^{2}\right)^{1 / 2} \quad \text { for } t \in[\tau, T],
$$

where $\Lambda=\sup _{M \times[0, T]}|H|(x, t)$.

The constant $C$ in (3-1) should depend on the Sobolev constant and the derivative of the volume form of the induced metric $g(t)$, which can be controlled by $|H|$ in our case. This can be seen from (2-2) and Michael and Simon's inequality [1973]

$$
\left(\int_{M}|f|^{n /(n-1)}\right)^{(n-1) / n} \leq c(n)\left(\int_{M}|\nabla f|+\int_{M}|H f|\right) .
$$

The following result is a corollary of Theorem 10 .

Proposition 11. Let $f$ be a nonnegative function satisfying

$$
\partial_{t} f \leq \Delta f+b f+\epsilon e^{-\gamma t} \quad \text { for } t \in[0, T],
$$

where $b$ and $\gamma$ are nonnegative constants and $\Delta$ is the Laplacian of the induced metric $g(t)$ of the mean curvature flow. Then

$$
f(t) \leq C(n, \Lambda, b, \tau)\left(\left(\int_{t-\tau}^{t} \int_{M} f^{2}\right)^{1 / 2}+\frac{\epsilon}{\gamma+b} e^{-\gamma t}\right) \quad \text { for } t \in[\tau, T],
$$

where $\Lambda=\sup _{M \times[0, T]}|H|(x, t)$.

Proof. We apply Theorem 10 to the function $\left|f+(\epsilon /(\gamma+b)) e^{-\gamma t}\right|$.

In the following, we need to use P. Topping's result [2008], which relates the upper bound of the induced diameter with the integral norm of the mean curvature.

Lemma 12. Let $n \geq 1$. Suppose that $M$ is an $n$-dimensional closed (compact, no boundary) connected manifold smoothly immersed in $\mathbb{R}^{n}$. Then its intrinsic diameter and its mean curvature $H$ are related by

$$
\operatorname{diam} \leq C(m) \int_{M}|H|^{m-1} d V .
$$

Proposition 13. Suppose that the solution $M_{t}$ satisfies the inequalities

$$
|A|(0) \leq \Lambda, \quad h \geq \gamma>0, \quad \int_{M}|\AA|(0)^{2} \leq \epsilon .
$$

Then there exists a $T=T(\Lambda)$ such that $M_{t}$ satisfies

$$
|A|(t) \leq 2 \Lambda \quad \text { and } \quad h \geq \gamma / 2 \text { for } t \in[0, T]
$$


and for fixed $\tau>0$ there exists some constant $C_{1}=C_{1}(n, \tau, \Lambda, v)$ such that

$$
\begin{aligned}
|H-h|^{2}(t) & \leq C_{1}(n, \tau, \Lambda, v) \epsilon^{1 / 2} \\
|\AA|(t) & \leq C_{1}(n, \tau, \Lambda, v) \epsilon^{1 / 2} \quad \text { for } t \in[\tau, T] .
\end{aligned}
$$

Proof. By Lemma 8, we have the inequality

$$
\partial_{t}|A| \leq \Delta|A|+|A|^{3}+h|A|^{2} .
$$

Using the inequality $|h|(t) \leq n \max _{M_{t}}|A|(t)$, the maximum principle implies

$$
|A|(t) \leq 2 \Lambda \text { for } t \in[0, T]
$$

where $T \leq c / \Lambda^{2}$ for a numerical constant $c>0$. By Lemma 9 we can calculate

$$
\begin{aligned}
& \frac{\partial}{\partial t} \int_{M}|\AA|^{2} d V_{t} \\
& \quad=\int_{M}-2|\nabla \AA|^{2}+2|A|^{2}|\AA|^{2}-2 h\left(\operatorname{tr}\left(\AA^{3}\right)+\frac{2}{n} H|\AA|^{2}\right)+|\AA|^{2} H(h-H) d V_{t} \\
& \quad \leq c(n) \Lambda^{2} \int_{M}|\AA|^{2},
\end{aligned}
$$

where we have used the facts that $|H|(t) \leq 2 n \Lambda$ and $|H-h|(t) \leq 4 n \Lambda$ for $t \in[0, T]$. Hence, we obtain

$$
\int_{M}|\stackrel{\AA}{2}|^{2} \leq e^{c(n) \Lambda^{2} t} \epsilon \quad \text { for } t \in[0, T] .
$$

By Lemma 9 again, $\partial_{t}|\AA| \leq \Delta|\AA|+c(n) \Lambda^{2}|\AA|$ for $t \in[0, T]$. Applying the parabolic Moser iteration, we have

$$
|\AA|(t) \leq C(n, \Lambda, \tau)\left(\int_{t-\tau}^{t} \int_{M}|\AA|^{2}\right)^{1 / 2} \leq C(n, \Lambda, \tau) \epsilon^{-1 / 2} \quad \text { for } t \in[\tau, T] .
$$

Now we estimate the change of $h(t)$. By its definition and Lemma 8, we obtain

$$
\left|\frac{d h}{d t}\right|=\left.\frac{1}{V_{t}}\left|\int_{M}(H-h)\right| A\right|^{2}+H^{2}(h-H)+h(H-h)^{2} \mid \leq c(n) \Lambda^{2},
$$

which implies

$$
h(t) \geq h(0)-c(n) \Lambda^{2} t \quad \text { for } t \in[0, T] .
$$

Finally, we estimate $|H-h|$. By the gradient estimates and (3-5) we have

$$
|\nabla A|(t) \leq C(\tau, \Lambda) \quad \text { and } \quad\left|\nabla^{2} A\right| \leq C(\tau, \Lambda) \quad \text { for } t \in[\tau, T] .
$$


Since $|\AA|$ is small, integrating by parts and Schwartz's inequality implies

$$
\begin{aligned}
\int_{M}|\nabla \AA|^{2} & \leq\left(\int_{M}|\AA|^{2}\right)^{1 / 2}\left(\int_{M}|\Delta \AA|^{2}\right)^{1 / 2} \\
& \leq C(\tau, \Lambda, v) e^{c(n)(\Lambda t)^{1 / 2}} \epsilon^{1 / 2} \quad \text { for } t \in[\tau, T] .
\end{aligned}
$$

By Lemma 9 and (3-8), $\partial_{t}|\nabla \AA| \leq \Delta|\nabla \AA|+C(\tau, \Lambda)|\AA|+\Lambda^{2}|\nabla \AA|$ for $t \in[\tau, T]$. By the parabolic Moser iteration and (3-7)(3-9), we have

$$
|\nabla \AA|(t) \leq C(\tau, \Lambda, v, n) \epsilon^{1 / 4} \quad \text { for } t \in[2 \tau, T] .
$$

Since $n \geq 2$, [Huisken 1984, Lemma 2.2] implies

$$
|\nabla \AA|^{2} \geq \frac{2(n-1)}{n(n+2)}|\nabla H|^{2} .
$$

Since the mean curvature is bounded, Lemma 12 implies diam $(t) \leq C(n, \Lambda, v)$ for $\in[0, T]$. Combining (3-10) with (3-11), we obtain for $t \in[2 \tau, T]$ that

$$
|H-h|(t) \leq \operatorname{diam}(t) \max |\nabla H| \leq C(n, \Lambda, v) \max |\nabla \AA| \leq C(\tau, \Lambda, v, n) \epsilon^{1 / 4} .
$$

The average of the mean curvature. Now we assume that the initial hypersurface has $h(0)>0$. The following lemma shows that $h(t)$ does not decay along the mean curvature flow if the traceless part of the second fundamental form is small.

Lemma 14. We have the following two implications:

If $\quad h(t)>0, \quad|\AA|(t) \leq \epsilon, \quad|H-h|^{2}(t) \leq \epsilon \quad$ for $t \in[0, T]$,

then $h(0) e^{-c(n) \epsilon t}-c(n) \epsilon^{3 / 2} t \leq h(t) \leq h(0)+2 \epsilon^{3 / 2} t \quad$ for $t \in[0, T]$.

If $\quad h(t)>0, \quad|\AA|(t) \leq \epsilon e^{-\gamma t}, \quad|H-h|^{2}(t) \leq \epsilon e^{-\gamma t} \quad$ for $t \in[0, T]$,

then $\quad h(0) e^{-c(n) \epsilon / \gamma}-\frac{c(n)}{\gamma} \epsilon^{3 / 2} e^{c(n) \epsilon / \gamma} \leq h(t) \leq h(0)+\frac{2}{3 \gamma} \epsilon^{3 / 2} \quad$ for $t \in[0, T]$.

Proof. From the definition of $h$, we have $V_{t} h=\int_{M} H d V_{t}$. Taking a derivative with respect to $t$, we have

$$
\begin{aligned}
V_{t} \frac{d h}{d t} & =\int_{M}(H-h)|A|^{2}+H^{2}(h-H)+h(H-h)^{2} \\
& =\int_{M}(H-h)|\AA|^{2}-\frac{n-1}{n} H^{2}(H-h)+h(H-h)^{2} \\
& =\int_{M}(H-h)|\AA|^{2}-\frac{n-1}{n}(H-h)^{3}-(1-2 / n) h(H-h)^{2},
\end{aligned}
$$

where we have used the fact that

$$
\int_{M} H^{2}(H-h)=\int_{M}(H-h)^{3}+2 h(H-h)^{2} .
$$


By the assumption of the first implication, we have

$$
-(1-2 / n) \epsilon h-c(n) \epsilon^{3 / 2} \leq \frac{d h}{d t} \leq 2 \epsilon^{3 / 2},
$$

which directly implies its conclusion. We can prove the second similarly.

The exponential decay. The key lemma of the proof of Theorem 1 shows that the traceless part of the second fundamental form decays exponentially if it is sufficiently small along the mean curvature flow.

Lemma 15. For any $\gamma>0$, there exists an $\epsilon_{0}=\epsilon_{0}(n, \gamma)$ such that if the solution $M_{t}$ satisfies the inequalities

$$
h(t) \geq \gamma>0, \quad|\AA|(t) \leq \epsilon, \quad|H-h|^{2}(t) \leq \epsilon
$$

for $t \in[0, T]$ and $\epsilon \in\left(0, \epsilon_{0}\right)$, then

$$
|\AA|(t) \leq e^{-\alpha t} \max _{M_{0}}|\AA|(0) \quad \text { for } t \in[0, T],
$$

where $\alpha=\gamma / \sqrt{2 n}$.

Proof. Recall that Lemma 9 implies

$$
\partial_{t}|\AA|^{2}=\Delta|\AA|^{2}-2|\nabla \AA|^{2}+2|A|^{2}|\AA|^{2}-2 h\left(\operatorname{tr}\left(\AA^{3}\right)+(2 / n) H|\AA|^{2}\right) .
$$

Note that

$$
\begin{aligned}
- & 2 h\left(\operatorname{tr}\left(\AA^{3}\right)+(2 / n) H|\AA|^{2}\right) \\
& =-2 H\left(\operatorname{tr}\left(\AA^{3}\right)+(2 / n) H|\AA|^{2}\right)+2(H-h)\left(\operatorname{tr}\left(\AA^{3}\right)+(2 / n) H|\AA|^{2}\right) \\
& \leq-(4 / n) H^{2}|\AA|^{2}+2 H|\AA|^{3}+2(H-h)\left(|\AA|^{3}+(2 / n) H|\AA|^{2}\right) \\
& \leq-(4 / n) H^{2}|\AA|^{2}+4\left(h|\AA|^{3}+h|H-h||\AA|^{2}+|H-h||\AA|^{3}+|H-h|^{2}|\AA|^{2}\right) .
\end{aligned}
$$

With this inequality, (3-14) implies

$$
\begin{aligned}
\partial_{t}|\AA|^{2} \leq \Delta|\AA|^{2}+2|A|^{2}|\AA|^{2}-(4 / n) H^{2}|\AA|^{2} \\
+4\left(h|\AA|^{3}+h|H-h||\AA|^{2}+|H-h||\AA|^{3}+|H-h|^{2}|\AA|^{2}\right) .
\end{aligned}
$$

Note that

$$
\begin{aligned}
2|A|^{2}|\AA|^{2} & -(4 / n) H^{2}|\AA|^{2} \\
& =-(2 / n) H^{2}|\AA|^{2}+2|\AA|^{4} \\
& =-(2 / n) h^{2}|\AA|^{2}+2\left(h|H-h||\AA|^{2}+(H-h)^{2}|\AA|^{2}+|\AA|^{4}\right),
\end{aligned}
$$


Combining the last two calculations with the assumptions, we have

$$
\begin{aligned}
\partial_{t}|\AA|^{2} \leq & \Delta|\AA|^{2}-(2 / n) h^{2}|\AA|^{2} \\
& +6\left(h|\AA|^{3}+h|H-h||\AA|^{2}+|H-h||\AA|^{3}+|H-h|^{2}|\AA|^{2}+|\AA|^{4}\right) \\
\leq & \Delta|\AA|^{2}-(1 / n)\left(h^{2}-c(n) \epsilon\right)|\AA|^{2} .
\end{aligned}
$$

Since $h(t) \geq \gamma$, we choose $\epsilon$ small enough that $(1 / n)\left(h^{2}-c(n) \epsilon\right)>(1 / 2 n) \gamma^{2}$. The lemma then follows from the maximum principle.

Proposition 11 and the proof of Proposition 13 then give the exponential decay of $|H-h|$.

Lemma 16. Suppose that the solution $M_{t}$ satisfies

$$
|A|(t) \leq \Lambda \quad \text { and } \quad|\AA|(t) \leq \epsilon e^{-\gamma t} \quad \text { for all } t \in[0, T] .
$$

Then for some $\tau>0$ we have

$$
|H-h|^{2}(t) \leq C(n, \tau, \Lambda) \epsilon e^{-\gamma t} \text { for } t \in[\tau, T] .
$$

Proof. By the gradient estimates and (3-16) we have

$$
|\nabla A|(t) \leq C(\tau, \Lambda) \quad \text { and } \quad\left|\nabla^{2} A\right| \leq C(\tau, \Lambda) \quad \text { for } t \in[\tau / 2, T] .
$$

Since $|\AA|$ is small, Schwartz's inequality implies

$$
\int_{M}|\nabla \AA|^{2} \leq\left(\int_{M}|\AA|^{2}\right)^{1 / 2}\left(\int_{M}|\Delta \AA|^{2}\right)^{1 / 2} \leq C(\tau, \Lambda, v) e^{-\gamma t} \epsilon
$$

By Lemma 9 and (3-17), $\nabla \AA$ satisfies the inequality

$$
\partial_{t}|\nabla \AA| \leq \Delta|\nabla \AA|+C(\tau, \Lambda)|\AA|+\Lambda^{2}|\nabla \AA| \text { for } t \in[\tau / 2, T] .
$$

By Proposition 11 and (3-16)(3-18), we have

$$
|\nabla \AA|(t) \leq C(\tau, \Lambda, v, n) e^{-\gamma t / 2} \epsilon^{1 / 2} \quad \text { for } t \in[\tau, T] .
$$

Since $n \geq 2$, [Huisken 1984, Lemma 2.2] implies

$$
|\nabla \AA|^{2} \geq \frac{2(n-1)}{n(n+2)}|\nabla H|^{2} .
$$

Combining (3-19) (3-20) with Lemma 12, we obtain

$$
\begin{aligned}
|H-h|(t) & \leq \operatorname{diam}(t) \max _{x \in M}|\nabla H| \leq c(n, \Lambda, v) \max _{x \in M}|\nabla \AA| l \\
& \leq C(n, \tau, \Lambda, v) e^{-\gamma t / 2} \epsilon^{1 / 2} \quad \text { for } t \in[\tau, T] .
\end{aligned}
$$


Proof of Theorem 1. The proof consists of several steps. In each step, we need to choose constants carefully.

Step 1. In this step, we give estimates near the initial time. Suppose the solution satisfies (1-2) at the initial time. Then by Proposition 13 there exists $T_{1}=T_{1}(\Lambda)$ such that

$$
|A|(t) \leq 2 \Lambda \quad \text { and } \quad h(t) \geq \gamma / 2 \quad \text { for } t \in\left[0, T_{1}\right],
$$

and for some $\tau<T_{1} / 4$ and some constant $C_{1}=C_{1}(n, \tau, \Lambda)$,

$$
|H-h|^{2}(t) \leq C_{1}(n, \tau, \Lambda) \epsilon^{1 / 2} \quad \text { and } \quad|\AA| \leq C_{1}(n, \tau, \Lambda) \epsilon^{1 / 2} \quad \text { for } t \in\left[\tau, T_{1}\right] .
$$

Now applying Lemma 15 for $t \in[\tau, T]$, we choose $\epsilon<\epsilon_{0}(n, \gamma)$ small so that

$$
|\AA|(t) \leq e^{-\alpha(t-\tau)}|\AA|(\tau) \leq C_{1}(\tau, \Lambda, D) \epsilon^{1 / 2} e^{-\alpha(t-\tau)} \quad \text { for } t \in\left[\tau, T_{1}\right],
$$

where $\alpha=\gamma /(4 \sqrt{2 n})$ and $C_{1} \epsilon^{1 / 2}<1$. By Lemma $16,|H-h|$ decays as

$$
|H-h|^{2}(t) \leq C_{2}(n, \tau, 2 \Lambda) \epsilon^{1 / 2} e^{-\alpha(t-\tau)} \quad \text { for } t \in\left[2 \tau, T_{1}\right]
$$

with $C_{2} \epsilon^{1 / 2}<1$. Set $C_{3}=\max \left\{C_{1}, C_{2}\right\}$. Now by Lemma $14, h(t)$ is bounded:

$$
h(t) \leq \sqrt{n}|A|(2 \tau)+\frac{2}{3 \alpha}\left(C_{3}\right)^{3 / 2} \epsilon^{3 / 4}<2 \sqrt{n} \Lambda+1 / 2 \quad \text { for } t \in\left[2 \tau, T_{1}\right],
$$

where we have chosen $\epsilon<\epsilon_{1}(\tau, \Lambda, \gamma)<\epsilon_{0}$ sufficiently small.

Step 2. In this step, we extend the solution to $\left[T_{1}, T_{1}+T_{2}\right]$ for some $T_{2}>0$. Recall these estimates for $t=T_{1}-\tau$ :

$$
\begin{aligned}
\gamma / 2 & \leq h(t)<2 \sqrt{n} \Lambda+1, & |\AA|(t) & \leq C_{3} \epsilon^{1 / 2} \epsilon^{-\alpha(t-\tau)}, \\
|A|(t) & \leq 2 \Lambda, & |H-h|^{2}(t) & \leq C_{3} \epsilon^{1 / 2} e^{-\alpha(t-\tau)} .
\end{aligned}
$$

An application of Proposition 13 for the initial time $t=T_{1}-\tau$ shows there exists a $T_{2}=T_{2}(2 \sqrt{n} \Lambda+3)>0$ such that

$$
\begin{aligned}
h(t) & \geq \gamma / 4, & |\AA| & \leq C_{4} \epsilon^{1 / 2} \epsilon^{-\alpha\left(T_{1}-\tau\right)}, \\
|A|(t) & \leq 4 \Lambda, & |H-h|^{2}(t) & \leq C_{4} \epsilon^{1 / 2} e^{-\alpha\left(T_{1}-\tau\right)} \quad \text { for } t \in\left[T_{1}, T_{1}+T_{2}\right],
\end{aligned}
$$

where $C_{4}=C_{1}(\tau, 2 \Lambda) C_{3}$. Now we choose $\epsilon$ small enough that

$$
C_{4} \epsilon^{1 / 2}<\epsilon_{0}(n, \gamma / 8)
$$

Then by Lemma 15 and the definition of $\alpha$, we have

$$
|\AA|(t) \leq e^{-\alpha(t-\tau)}|\AA|_{\infty}(\tau) \leq C_{1} \epsilon^{1 / 2} e^{-\alpha(t-\tau)} \quad \text { for } t \in\left[\tau, T_{1}+T_{2}\right] .
$$

By Lemma 14, we can choose $\epsilon$ small enough that

$$
h(t) \leq h(2 \tau)+2\left(C_{4} \epsilon^{1 / 2} e^{-\alpha\left(T_{1}-\tau\right)}\right)^{3 / 2}\left(T_{2}+T_{1}\right)<2 \sqrt{n} \Lambda+1 \quad \text { for } t \in\left[T_{1}, T_{1}+T_{2}\right],
$$


and therefore

$$
|A|(t) \leq|\AA|+|H-h|+h \leq 2 \sqrt{n} \Lambda+3 \text { for } t \in\left[T_{1}, T_{1}+T_{2}\right] .
$$

By Lemma 16,

$$
|H-h|^{2} \leq C_{2}(n, \tau, 2 \sqrt{n} \Lambda+3) C_{1} \epsilon^{1 / 2} e^{-\alpha(t-\tau)} \quad \text { for } t \in\left[2 \tau, T_{1}+T_{2}\right] .
$$

Set $C_{6}=\max \left\{C_{2}(n, \tau, 2 \sqrt{n} \Lambda+3) C_{1}, C_{1}\right\}$. Choose $\epsilon$ small enough that $C_{6} \epsilon^{1 / 2}<1$. Then by Lemma 14 we can choose $\epsilon$ small enough that

$$
h(t) \geq h(\tau) e^{-c(n) / \alpha C_{6} \epsilon^{1 / 2}}-(c(n) / \alpha)\left(C_{6} \epsilon^{1 / 2}\right)^{3 / 2} e^{c(n) / \alpha\left(C_{6} \epsilon^{1 / 2}\right)} \geq \gamma / 3
$$

for $t \in\left[T_{1}, T_{1}+T_{2}\right]$, and

$$
h(t) \leq h(2 \tau)+\frac{2}{3 \alpha}\left(C_{6} \epsilon^{1 / 2}\right)^{3 / 2}<2 \sqrt{n} \Lambda+1 \quad \text { for } t \in\left[2 \tau, T_{1}+T_{2}\right] .
$$

Claim 17 (Step 3). If the estimates

$$
\begin{aligned}
\gamma / 3 & \leq h(t)<2 \sqrt{n} \Lambda+1, \\
|\AA|(t) & \leq C_{6} \epsilon^{1 / 2} \epsilon^{-\alpha(t-\tau)}, \quad|H-h|^{2}(t) \leq C_{6} \epsilon^{1 / 2} e^{-\alpha(t-\tau)}
\end{aligned}
$$

hold for $t \in[2 \tau, S]$ and $S \geq T_{1}+T_{2}$, then they also hold for $t \in\left[2 \tau, S+T_{2}\right]$.

Proof. The assumptions imply that $|A|$ is bounded, that is,

$$
|A|(t) \leq|\AA|(t)+|H-h|(t)+h(t) \leq \Lambda_{1}=2 \sqrt{n} \Lambda+3 \quad \text { for } t \in[\tau, S] .
$$

Now we apply Proposition 13 for $t=S-\tau$. By the definition of $T_{2}$ we have

$$
\begin{aligned}
h(t) & \geq \gamma / 6, \quad|\AA|(t) & \leq C_{7} \epsilon^{1 / 2} \epsilon^{-\alpha(S-\tau)}, \\
|A|(t) & \leq 2 \Lambda_{1}, \quad|H-h|(t) & \leq C_{7} \epsilon^{1 / 2} e^{-\alpha(S-\tau)} \quad \text { for } t \in\left[S, S+T_{2}\right],
\end{aligned}
$$

where $C_{7}=C_{1}\left(n, \tau, 2 \Lambda_{1}\right) C_{6}$. Lemma 14 implies that if we choose $\epsilon<\epsilon_{2}$ small enough,

$$
h(t) \geq h(S) e^{-c(n) C_{7} \epsilon^{1 / 2} T_{2}}-c(n)\left(C_{7} \epsilon^{1 / 2}\right)^{3 / 2} T_{2} \geq \gamma / 4 \quad \text { for } t \in\left[S, S+T_{2}\right] .
$$

By Lemma 15 we have

$$
|\AA|(t) \leq e^{-\alpha(t-\tau)}|\AA|_{\infty}(\tau) \leq C_{1} \epsilon^{1 / 2} e^{-\alpha(t-\tau)} \quad \text { for } t \in\left[\tau, S+T_{2}\right] .
$$

By Lemma 16 and the definition of $C_{6}$, we have

$$
\begin{aligned}
|H-h|^{2} & \leq C_{2}(n, \tau, 2 \sqrt{n} \Lambda+3) C_{1} \epsilon^{1 / 2} e^{-\alpha(t-\tau)} \\
& \leq C_{6} \epsilon^{1 / 2} e^{-\alpha(t-\tau)} \quad \text { for } t \in\left[2 \tau, S+T_{2}\right] .
\end{aligned}
$$

By Lemma 14, we have

$$
h(t) \leq h(2 \tau)+\frac{2}{3 \alpha}\left(C_{6} \epsilon^{1 / 2}\right)^{3 / 2}<2 \sqrt{n} \Lambda+1 \quad \text { for } t \in\left[2 \tau, S+T_{2}\right],
$$


and therefore

$$
|A|(t) \leq|\AA|+|H-h|+h \leq 2 \sqrt{n} \Lambda+3 \text { for } t \in\left[S, S+T_{2}\right] .
$$

Then by Lemma 14

$$
\begin{aligned}
h(t) & \geq h(\tau) e^{-(c(n) / \alpha) C_{6} \epsilon^{1 / 2}}-(c(n) / \alpha)\left(C_{6} \epsilon^{1 / 2}\right)^{3 / 2} e^{(c(n) / \alpha) C_{6} \epsilon^{1 / 2}} \\
& \geq \gamma / 3 \text { for } t \in\left[T_{1}, T_{1}+T_{2}\right] .
\end{aligned}
$$

Step 4. By Claim 17, the flow has long time existence and converges exponentially fast to a umbilic hypersurface with constant mean curvature, which is a round sphere since $M$ is compact and orientable.

Proof of Corollary 4. We follow the arguments in [Huisken and Yau 1996]. Let $M$ be a compact smooth hypersurface in $B_{\sigma}$ for some positive constants $\delta, \sigma$ and $B_{i}$ for $i=1, \ldots, 4$. Then the gradient of the second fundamental form can be controlled by [Huisken 1984, Lemma 2.2], that is,

$$
|\nabla A|^{2} \leq \frac{3 n}{2 n-2}|\nabla \AA|^{2} \leq \frac{c\left(B_{3}, n\right)}{\sigma^{4+2 \delta}} .
$$

Following the arguments in [Huisken and Yau 1996, Propositions 2.1 and 2.2], we can estimate the mean curvature and the second fundamental form by

$$
|H-n / \sigma| \leq c\left(B_{i}, n\right) / \sigma^{1+\delta} \quad \text { and } \quad|A| \leq c\left(B_{i}, n\right) / \sigma .
$$

Now we rescale the hypersurface by $\widetilde{M}=\sigma^{-1} M$, and the above estimates can be reduced to

$$
\tilde{V} \leq B_{4}, \quad|\tilde{A}|_{\tilde{g}} \leq c\left(B_{i}, n\right), \quad|\tilde{H}-n| \leq \frac{c\left(B_{i}, n\right)}{\sigma^{\delta}}, \quad \int_{\tilde{M}}|\tilde{A}|_{\tilde{g}}^{2} \leq \frac{c\left(B_{i}, n\right)}{\sigma^{2 \delta}} .
$$

Therefore, the assumptions of Theorem 1 are satisfied if $\sigma$ is sufficiently large. This proves the first part of the corollary. The second follows similarly.

\section{Proof of Theorem 5}

In this section, we extend the usual $\epsilon$-regularity of the mean curvature flow to the case of $L^{2}$ norm of the traceless second fundamental form. As stated in the introduction, we prove that under the assumptions of Theorem 5, the flow will satisfy the condition (1-2) of Theorem 1 after a short time. More precisely:

Proposition 18. Let $M_{0} \subset \mathbb{R}^{3}$ be a compact smooth surface. For any $v_{0}, \Lambda, \gamma>$ 0 with $\gamma<\Lambda$, there exists a constant $\epsilon_{0}=\epsilon_{0}\left(v_{0}, \Lambda, \gamma\right)>0$ such that for any $\epsilon \in\left(0, \epsilon_{0}\right)$, any solution of the mean curvature flow satisfying

$$
0<\gamma<h<\Lambda, \quad V_{0} \geq v_{0}>0, \quad \int_{M}|H-h|^{2}<\epsilon, \quad \int_{M}|\AA|^{2} \leq \epsilon,
$$


there exists a $T=T\left(\Lambda, \gamma, v_{0}\right)$ such that

(4-2) $\frac{1}{2} \gamma \leq h \leq 2 \Lambda, \quad V(T) \geq \frac{1}{2} v_{0}, \quad|A|(T) \leq C\left(\Lambda, \gamma, v_{0}\right), \quad \int_{M}|\AA|^{2}(T) \leq 2 \epsilon$.

The proof of this proposition is a little long but the argument is fairly standard. Roughly, we use the $\epsilon$-regularity to control the $L^{\infty}$ norm of the second fundamental form by some integral norms, which can be easily bounded along the flow. In Section 4A, we estimate the integral norms for a short time. In Section 4B, we will use the integral norms to control the $L^{\infty}$ norm of the second fundamental form. In Section $4 \mathrm{C}$, we complete the proof.

4A. The $L^{2}$ norm of traceless second fundamental form. The next two lemmas show that the $L^{4}$ norms of $|\AA|$ and $H-h$ remain small for a short time under some integral conditions.

Lemma 19. Suppose exists a numerical constant $\epsilon_{0}>0$ such that if

$$
\int_{M}|\AA|^{2} \leq \epsilon \quad \text { and } \quad \int_{M}|H-h|^{2} \leq \epsilon \quad \text { for } t \in[0, T]
$$

whenever $\epsilon \in\left(0, \epsilon_{0}\right)$, we have the inequality

$$
\int_{M}|\AA|^{2}(t)+\frac{1}{n} \int_{0}^{t} \int_{M}\left(|\nabla \AA|^{2}+h^{2}|A|^{2}+|\AA|^{4}\right) \leq \int_{M}|\AA|^{2}(0) \quad \text { for } t \in[0, T] \text {. }
$$

Proof. By the inequality (3-15), $|\AA|$ satisfies

$$
\begin{aligned}
& \frac{\partial}{\partial t} \int_{M}|\AA|^{2}+\int_{M} 2|\nabla \AA|^{2} d V_{t} \\
& \leq-\frac{2}{n} \int_{M} h^{2}|\AA|^{2} \\
& \quad+5\left(h|\AA|^{3}+h|H-h||\AA|^{2}+|H-h||\AA|^{3}+|H-h|^{2}|\AA|^{2}+|\AA|^{4}\right) .
\end{aligned}
$$

Note that Schwarz's inequality implies

$$
\begin{aligned}
\int_{M}\left(h|\AA|^{3}+h|H-h||\AA|^{2}\right. & \left.+|H-h||\AA|^{3}+|H-h|^{2}|\AA|^{2}+|\AA|^{4}\right) \\
& \leq \int_{M}\left(\epsilon^{\prime} h^{2}|\AA|^{2}+c\left(\epsilon^{\prime}\right)|\AA|^{4}+c\left(\epsilon^{\prime}\right)|H-h|^{2}|\AA|^{2}\right) .
\end{aligned}
$$

Now applying the Sobolev inequality, we obtain

$$
\begin{aligned}
\int_{M}|\AA|^{4} \leq c\left(\int_{M}|\AA||\nabla \AA|+|H||\AA|^{2}\right)^{2} & \\
& \leq c \int_{M}|\AA|^{2}\left(\int_{M}|\nabla \AA|^{2}+\int_{M}|H-h|^{2}|\AA|^{2}+\int_{M} h^{2}|\AA|^{2}\right),
\end{aligned}
$$


and

$$
\begin{aligned}
\int_{M} \mid H & -\left.h\right|^{2}|\AA|^{2} \\
& \leq c\left(\int_{M}|\nabla H||\AA|+|H-h||\nabla \AA|+|H(H-h)||\AA|\right)^{2} \\
& \leq c \int_{M}|\nabla \AA|^{2}\left(\int_{M}|\AA|^{2}+\int_{M}|H-h|^{2}\right)+c \int_{M}|H-h|^{2} \int_{M} H^{2}|\AA|^{2} \\
& \leq c \int_{M}|\nabla \AA|^{2}\left(\int_{M}|\AA|^{2}+\int_{M}|H-h|^{2}\right) \\
& \quad+c \int_{M}|H-h|^{2}\left(\int_{M}|H-h|^{2}|\AA|^{2}+\int_{M} h^{2}|\AA|^{2}\right) .
\end{aligned}
$$

Combining the inequalities (4-4)-(4-7), we can choose $\epsilon$ small to arrive at

$$
\frac{\partial}{\partial t} \int_{M}|\AA|^{2}+\int_{M}\left(|\nabla \AA|^{2}+|\AA|^{4}\right)+\frac{1}{n} \int_{M} h^{2}|\AA|^{2} \leq 0 \quad \text { for } t \in[0, T] .
$$

Integrating from 0 to $t$, we obtain

$$
\int_{M}|\AA|^{2}(t)+\frac{1}{n} \int_{0}^{t} \int_{M}\left(|\nabla \AA|^{2}+h^{2}|A|^{2}+|\AA|^{4}\right) \leq \int_{M}|\AA|^{2}(0) .
$$

Lemma 20. There exists a numerical constant $\epsilon_{0}>0$ such that if

$$
\int_{M}|\AA|^{2} \leq \epsilon \quad \text { and } \quad \int_{M}|H-h|^{2} \leq \epsilon \quad \text { for } t \in[0, T]
$$

whenever $\epsilon \in\left(0, \epsilon_{0}\right)$, then

$$
\begin{aligned}
\int_{M}(H-h)^{2}(t)+\int_{0}^{t} & \left(\int_{M}|\nabla H|^{2}+\int_{M}|H-h|^{4}\right) \\
& \leq 2 e^{6 \int_{0}^{t} h^{2}}\left(\int_{M}(H-h)^{2}(0)+\int_{M}|\AA|^{2}(0)\right) \quad \text { for } t \in[0, T] .
\end{aligned}
$$

Proof. By Lemma 8, $H-h$ satisfies the evolution equation

$$
\begin{aligned}
\frac{\partial}{\partial t} & \int_{M}(H-h)^{2} \\
& =\int_{M} 2(H-h)\left(\Delta H+2(H-h)|A|^{2}\right)-H(H-h)^{3} \\
& =-\int_{M} 2|\nabla H|^{2}+\int_{M} 4(H-h)^{2}\left(|\AA|^{2}+\frac{1}{2} H^{2}\right)-(H-h)^{4}-h(H-h)^{3} \\
& =-\int_{M} 2|\nabla H|^{2}+\int_{M} 4(H-h)^{2}|\AA|^{2}+(H-h)^{4}+3 h(H-h)^{3}+2 h^{2}(H-h)^{2} \\
& \leq-\int_{M} 2|\nabla H|^{2}+5 \int_{M}\left(|\AA|^{4}+(H-h)^{4}+h^{2}(H-h)^{2}\right) .
\end{aligned}
$$


We apply the Sobolev inequality to find

$$
\begin{aligned}
\int_{M}(H-h)^{4} & \leq c\left(\int_{M}|H-h||\nabla H|+|H||H-h|^{2}\right)^{2} \\
\leq c \int_{M}(H-h)^{2} \int_{M}|\nabla H|^{2} & \\
& \quad+c \int_{M}(H-h)^{2}\left(\int_{M}(H-h)^{4}+\int_{M} h^{2}(H-h)^{2}\right),
\end{aligned}
$$

where $c$ is a numerical constant. Combining the previous two inequalities, we choose $\epsilon<1 / c$ small to arrive at

$$
\frac{\partial}{\partial t} \int_{M}(H-h)^{2}+\int_{M}|\nabla H|^{2}+|H-h|^{4} \leq 6 \int_{M}|\AA|^{4}+h^{2}(H-h)^{2} .
$$

Integrating from 0 to $t$ and applying (4-3) yields the claimed inequality.

The next lemma shows that the volume and the average of the mean curvature change slowly near the initial time.

Lemma 21. The volume $V_{t}$ and the average of the mean curvature $h(t)$ satisfy

$$
V_{t} \geq V_{0}-E(t) \quad \text { and } \quad|h(t)-h(0)| \leq \frac{1}{V_{0}-E(t)} E(t) \quad \text { for } t \in[0, T],
$$

where

$$
E(t)=\int_{0}^{t} \int_{M}(H-h)^{2}+|\AA|^{4}+(H-h)^{4} .
$$

Proof. By (3-12) and the assumption $n=2$, we have

$$
\begin{aligned}
V_{t} \frac{d}{d t} h(t) & =\int_{M}(H-h)|\AA|^{2}-\frac{1}{2}(H-h)^{3} \\
& \leq\left(\int_{M}(H-h)^{2}\right)^{1 / 2}\left(\int_{M}|\AA|^{4}+\int_{M}(H-h)^{4}\right)^{1 / 2} \\
& \leq \int_{M}(H-h)^{2}+|\AA|^{4}+(H-h)^{4}
\end{aligned}
$$

since the volume satisfies $d V_{t} / d t=-\int_{M}(H-h)^{2}$. Integrating from 0 to $t$, we obtain

$$
V_{t} \geq V_{0}-E(t)
$$

Combining this with (4-9), we have

$$
|h(t)-h(0)| \leq \frac{1}{V_{0}-E(t)} E(t) \quad \text { for } t \in[0, T] .
$$


4B. The $L^{\infty}$ norm of the second fundamental form. Now we prove that under some integral conditions, the $L^{\infty}$ norm of $|\AA|$ will be bounded after a short time.

Proposition 22. For any given $\Lambda, v_{0}, T>0$, there exists an $\epsilon_{0}=\epsilon\left(\Lambda, v_{0}, T\right)>0$ such that if

$$
0<h(t) \leq \Lambda, \quad E(t) \leq \epsilon, \quad \int_{M}(H-h)^{2}(t) \leq 1 \quad \text { for } t \in[0, T],
$$

and $V_{0} \geq v_{0}$, where $E(t)$ is given by (4-8), then

$$
\sup _{t \in[T / 2, T]}|A|^{2} \leq C\left(\Lambda, v_{0}, T\right) .
$$

We prove this proposition by following the argument in [Ecker 1995]. Let $\eta(t): \mathbb{R} \rightarrow \mathbb{R}$ be any nonnegative smooth function with $\eta(0)=0$. For simplicity, we define $c_{\eta}$ as a operator satisfying

$$
c_{\eta} f=\eta^{\prime} \cdot\left(f \eta^{-1}\right)
$$

for any smooth function of the form $f=g \eta$ with $g \in C^{\infty}(M \times[0, \infty))$.

Lemma 23. For any $p \geq 1$, we have the inequalities

$$
\begin{gathered}
\left(\int_{0}^{T} \int_{M}\left(|\AA|^{2} \eta^{s}\right)^{4 p}\right)^{1 / 2} \leq c p \int_{0}^{T} \int_{M}\left(|\AA|^{2}+H^{2}+h^{2}+s c_{\eta}\right)\left(|\AA|^{2} \eta^{s}\right)^{2 p} \\
\left(\int_{0}^{T} \int_{M}\left((H-h)^{2} \eta^{s}\right)^{4 p}\right)^{1 / 2} \leq c p \int_{0}^{T} \int_{M}\left(|A|^{2}+\left|h^{\prime}\right| \eta^{s p}+s c_{\eta}\right)\left((H-h)^{2} \eta^{s}\right)^{2 p} \\
+c p \int_{0}^{T} \int_{M}\left|h^{\prime}\right| \eta^{s p}(H-h)^{2} .
\end{gathered}
$$

Here $c$ is a numerical constant and $c_{\eta}$ is defined by (4-12).

Proof. By Lemma 9, А satisfies

$$
\begin{aligned}
\partial_{t}|\AA|^{2} & \leq \Delta|\AA|^{2}-2|\nabla \AA|^{2}+2|A|^{2}|\AA|^{2}+2 h\left(|\AA|^{3}+|H||\AA|^{2}\right) \\
& \leq \Delta|\AA|^{2}-2|\nabla \AA|^{2}+3|\AA|^{4}+3 H^{2}|\AA|^{2}+2 h^{2}|\AA|^{2},
\end{aligned}
$$

Now we compute the evolution equation of $|\AA|^{2 p}$ to be

$$
\partial_{t}|\AA|^{2 p} \leq \Delta\left(|\AA|^{2 p}\right)+3 p\left(|\AA|^{2}+H^{2}+h^{2}\right)|\AA|^{2 p} .
$$

Hence $f=\left(|\AA|^{2} \eta^{s}\right)^{p}$ satisfies $\partial_{t} f \leq \Delta f+g f$, where $g=3 p\left(|\AA|^{2}+H^{2}+h^{2}+s c_{\eta}\right)$. Integrating $f^{2}$, we get

$$
\frac{d}{d t} \int_{M} f^{2} \leq \int_{M}\left(-2|\nabla f|^{2}+(2 g+H(h-H)) f^{2}\right) .
$$


Integrating from 0 to $T$ yields

$$
\sup _{t \in[0, T]} \int_{M} f^{2}+2 \int_{0}^{T} \int_{M}|\nabla f|^{2} \leq \int_{0}^{T} \int_{M}(2 g+H(h-H)) f^{2} .
$$

On the other hand, applying the Sobolev inequality and Schwartz's inequality gives

$$
\begin{aligned}
\int_{0}^{T} \int_{M} f^{4} & \leq c \int_{0}^{T}\left(\int_{M} 2|f||\nabla f|+|H| f^{2}\right)^{2} \\
& \leq c \sup _{t \in[0, T]} \int_{M} f^{2} \cdot \int_{0}^{T} \int_{M}\left(|\nabla f|^{2}+\left|H^{2}\right| f^{2}\right),
\end{aligned}
$$

where $c$ is a numerical constant. Combining these two inequalities, we obtain

$$
\left(\int_{0}^{T} \int_{M} f^{4}\right)^{1 / 2} \leq c \int_{0}^{T} \int_{M} g f^{2}
$$

which implies the lemma's first inequality.

Now we prove the second inequality. By Lemma $8, H-h$ satisfies

$$
\partial_{t}(H-h)=\Delta(H-h)+(H-h)|A|^{2}-h^{\prime} .
$$

Hence, for any $p \geq 1$, we have

$$
\begin{aligned}
\partial_{t}(H-h)^{2 p} & \leq \Delta(H-h)^{2 p}+2 p(H-h)^{2 p}|A|^{2}-2 p(H-h)^{2 p-1} h^{\prime} \\
& \leq \Delta(H-h)^{2 p}+\left(2 p|A|^{2}+2 p\left|h^{\prime}\right|\right)(H-h)^{2 p}+2 p\left|h^{\prime}\right||H-h|,
\end{aligned}
$$

where we have used that $|H-h|^{2 p-1} \leq|H-h|+|H-h|^{2 p}$ for $p \geq 1$. The function $f=\left((H-h)^{2} \eta^{s}\right)^{p}$ satisfies

$$
\partial_{t} f \leq \Delta f+a f+b
$$

where $a=2 p|A|^{2}+2 p\left|h^{\prime}\right| \eta^{s p}+s p c_{\eta}$ and $b=2 p\left|h^{\prime}\right||H-h| \eta^{s p}$. Integrating the inequality above, we have

$$
\begin{aligned}
\frac{\partial}{\partial t} \int_{M} f^{2} & \leq \int_{M}-2|\nabla f|^{2}+(a+H(h-H)) f^{2}+b f \\
& \leq \int_{M}-2|\nabla f|^{2}+\left(a+h^{2}+p\left|h^{\prime}\right| \eta^{s p}\right) f^{2}+p \eta^{s p}\left|h^{\prime}\right|(H-h)^{2},
\end{aligned}
$$

where we have used the fact that $b f \leq p\left|h^{\prime}\right| \eta^{s p}\left((H-h)^{2}+f^{2}\right)$. Integrating from 0 to $T$, we have

$$
\begin{aligned}
\sup _{t \in[0, T]} \int_{M} f^{2}+2 \int_{0}^{T} & \int_{M}|\nabla f|^{2} \\
& \leq \int_{0}^{T} \int_{M}\left(a+h^{2}+p\left|h^{\prime}\right| \eta^{s p}\right) f^{2}+p\left|h^{\prime}\right| \eta^{s p}(H-h)^{2}
\end{aligned}
$$


Therefore, we have

$$
\left(\int_{0}^{T} \int_{M} f^{4}\right)^{1 / 2} \leq c \int_{0}^{T} \int_{M}\left(a+h^{2}+p\left|h^{\prime}\right| \eta^{s p}\right) f^{2}+p\left|h^{\prime}\right| \eta^{s p}(H-h)^{2} .
$$

Lemma 24. There exists a constant $\epsilon_{0}=\epsilon_{0}\left(v_{0}\right)>0$ such that if $E(T) \in\left(0, \epsilon_{0}\right)$ and

$$
\sup _{t \in[0, T]} \int_{M}(H-h)^{2} \leq 1
$$

then

$$
\int_{T / 4}^{T} \int_{M}|\AA|^{8}+\int_{T / 4}^{T} \int_{M}|H-h|^{8} \leq c\left(1+1 / v_{0}\right)^{2}\left(\Lambda^{2}+1 / T+1\right)^{2} E(T)^{2},
$$

and

$$
\sup _{t \in[T / 4, T]}\left|h^{\prime}\right| \leq c / v_{0}
$$

Proof. Lemma 23 with $p=1$ and $s=4$ implies

$$
\begin{aligned}
&\left(\int_{0}^{T} \int_{M}\left(|\AA|^{2} \eta^{4}\right)^{4}\right)^{1 / 2} \leq c \int_{0}^{T} \int_{M}\left(|\AA|^{2}+(H-h)^{2}+h^{2}+c_{\eta}\right)\left(|\AA|^{2} \eta^{4}\right)^{2} \\
& \leq c\left(\int_{0}^{T} \int_{M}\left(|\AA|^{2} \eta^{4}\right)^{4}\right)^{1 / 2}\left(\left(\int_{0}^{T} \int_{M}|\AA|^{4}\right)^{1 / 2}\right.\left.+\left(\int_{0}^{T} \int_{M}(H-h)^{4}\right)^{1 / 2}\right) \\
&+c \int_{0}^{T} \int_{M}\left(c_{\eta}+\Lambda^{2}\right)\left(|\AA|^{2} \eta^{4}\right)^{2} .
\end{aligned}
$$

If $E(T)$ is sufficiently small, we obtain

$$
\left(\int_{0}^{T} \int_{M}\left(|\AA|^{2} \eta^{4}\right)^{4}\right)^{1 / 2} \leq c \int_{0}^{T} \int_{M}\left(c_{\eta}+\Lambda^{2}\right)\left(|\AA|^{2} \eta^{4}\right)^{2} .
$$

Now we take

$$
\eta(t)=\eta_{0}\left(\frac{t-T / 8}{T / 4-T / 8}\right)
$$

where $\eta_{0}$ is a cutoff function in $C^{\infty}(\mathbb{R}, \mathbb{R})$ such that $0 \leq \eta_{0} \leq 1,\left|\eta_{0}^{\prime}\right| \leq 2$, and

$$
\eta_{0}(t)= \begin{cases}0 & \text { if } t \leq 0 \\ 1 & \text { if } t \geq 1\end{cases}
$$

We have

$$
\left(\int_{T / 4}^{T} \int_{M}|\AA|^{8}\right)^{1 / 2} \leq c\left(\Lambda^{2}+\frac{16}{T}\right) \int_{T / 8}^{T} \int_{M}|\AA|^{4} \leq c\left(\Lambda^{2}+\frac{16}{T}\right) E(T),
$$


where we have used the fact that

$$
c_{\eta}\left(|\AA|^{2} \eta^{4}\right)^{2} \leq \frac{16}{T}|\AA|^{4} \eta^{7} \quad \text { for } t \in[0, T] .
$$

Similarly, by Lemma 23 we have

$$
\begin{aligned}
& \left(\int_{0}^{T} \int_{M}\left(|H-h|^{2} \eta^{4}\right)^{4}\right)^{1 / 2} \\
& \leq\left(\int_{0}^{T} \int_{M}\left(|H-h|^{2} \eta^{4}\right)^{4}\right)^{1 / 2}\left(\left(\int_{0}^{T} \int_{M}|\AA|^{4}\right)^{1 / 2}+\left(\int_{0}^{T} \int_{M}(H-h)^{4}\right)^{1 / 2}\right) \\
& \quad+c \int_{0}^{T} \int_{M}\left(\Lambda^{2}+c_{\eta}+\sup _{t \in[0, T]}\left|h^{\prime} \eta^{4}\right|\right)\left(|H-h|^{2} \eta^{4}\right)^{2} \\
& \quad+c \sup _{t \in[0, T]}\left|h^{\prime} \eta^{4}\right| \int_{0}^{T} \int_{M}|H-h|^{2} .
\end{aligned}
$$

Hence, if $E(T)$ is sufficiently small we have

$$
\begin{aligned}
& \left(\int_{0}^{T} \int_{M}\left(|H-h|^{2} \eta^{4}\right)^{4}\right)^{1 / 2} \\
& \leq c\left(\Lambda^{2}+1 / T+\sup _{t \in[0, T]}\left|h^{\prime} \eta^{4}\right|\right) \int_{0}^{T} \int_{M}|H-h|^{4}+c \sup _{t \in[0, T]}\left|h^{\prime} \eta^{4}\right| \int_{0}^{T} \int_{M}|H-h|^{2} .
\end{aligned}
$$

Here, the cutoff function $\eta$ is as in (4-17), so $c_{\eta}$ can be estimated by (4-20).

Next, we estimate $\sup _{t \in[0, T]}\left|h^{\prime} \eta^{4}\right|$. In fact, (4-9) implies

$$
V_{t}\left|h^{\prime}\right| \leq e(t):=\int_{M}(H-h)^{2}+|\AA|^{4}+(H-h)^{4} .
$$

Taking the cutoff function $\eta$ as in (4-17), the inequality (4-15) implies

$$
\begin{aligned}
& \sup _{t \in[0, T]} \int_{M}\left((H-h)^{2} \eta^{4}\right)^{2} \\
& \leq\left(\int_{0}^{T} \int_{M}\left(|H-h|^{2} \eta^{4}\right)^{4}\right)^{1 / 2}\left(\left(\int_{0}^{T} \int_{M}|\AA|^{4}\right)^{1 / 2}+\left(\int_{0}^{T} \int_{M}(H-h)^{4}\right)^{1 / 2}\right) \\
& +c\left(\Lambda^{2}+1 / T+\sup _{t \in[0, T]}\left|h^{\prime} \eta^{4}\right|\right) \int_{0}^{T} \int_{M}|H-h|^{4} \\
& \quad+c \sup _{t \in[0, T]}\left|h^{\prime} \eta^{4}\right| \int_{0}^{T} \int_{M}|H-h|^{2} \\
& \leq c \sup _{t \in[0, T]}\left|h^{\prime} \eta^{4}\right|\left(E(T)+E(T)^{3 / 2}\right)+c\left(\Lambda^{2}+1 / T\right)\left(E(T)+E(T)^{3 / 2}\right),
\end{aligned}
$$


where we have used the inequality (4-21). By (4-14), we have

$$
\begin{aligned}
& \text { (4-24) } \sup _{t \in[0, T]} \int_{M}\left(|\AA|^{2} \eta^{4}\right)^{2} \\
& \quad \leq\left(\int_{0}^{T} \int_{M}\left(|\AA|^{2} \eta^{4}\right)^{4}\right)^{1 / 2}\left(\left(\int_{0}^{T} \int_{M}|\AA|^{4}\right)^{1 / 2}+\left(\int_{0}^{T} \int_{M}(H-h)^{4}\right)^{1 / 2}\right) \\
& \quad+c\left(\Lambda^{2}+1 / T\right) \int_{0}^{T} \int_{M}(H-h)^{4} \\
& \leq c\left(\Lambda^{2}+1 / T\right)\left(E(T)+E(T)^{3 / 2}\right) .
\end{aligned}
$$

Combining the inequalities (4-22)-(4-24) with (4-10) yields

$$
\begin{aligned}
\left(V_{0}-E(T)\right) \sup _{t \in[0, T]}\left|h^{\prime} \eta^{4}\right| & \leq c \sup _{t \in[0, T]}\left|h^{\prime} \eta^{4}\right|\left(E(T)+E(T)^{3 / 2}\right) \\
& +c\left(\Lambda^{2}+1 / T\right)\left(E(T)+E(T)^{3 / 2}\right)+\sup _{t \in[0, T]} \int_{M}(H-h)^{2} .
\end{aligned}
$$

If $E(T)$ is sufficiently small, we have

$$
\sup _{t \in[0, T]}\left|h^{\prime} \eta^{4}\right| \leq \frac{c}{v_{0}}\left(\left(\Lambda^{2}+16 / T\right) E(T)+1\right)
$$

which implies (4-16). Combining (4-21) with (4-25), we have

$$
\left(\int_{T / 4}^{T} \int_{M}|H-h|^{8}\right)^{1 / 2} \leq c\left(1+1 / v_{0}\right)\left(\Lambda^{2}+1 / T+1\right) E(T) .
$$

The lemma immediately from this and (4-19).

Proof of Proposition 22. Now Hölder's inequality implies

$$
\begin{aligned}
& p \int_{0}^{T} \int_{M}|\AA|^{2}\left(|\AA|^{2} \eta^{4}\right)^{2 p} \\
& \leq p\left(\int_{0}^{T} \int_{\eta>0}|\AA|^{8}\right)^{1 / 4}\left(\int_{0}^{T} \int_{M}\left(|\AA|^{2} \eta^{4}\right)^{8 / 3 p}\right)^{3 / 4} \\
& \leq p\left(\int_{0}^{T} \int_{\eta>0}|\AA|^{8}\right)^{1 / 4}\left(\int_{0}^{T} \int_{M}\left(|\AA|^{2} \eta^{4}\right)^{4 p}\right)^{1 / 4}\left(\int_{0}^{T} \int_{M}\left(|\AA|^{2} \eta^{4}\right)^{2 p}\right)^{1 / 2} \\
& \leq\left(\int_{0}^{T} \int_{\eta>0}|\AA|^{8}\right)^{1 / 4}\left(\left(\int_{0}^{T} \int_{M}\left(|\AA|^{2} \eta^{4}\right)^{4 p}\right)^{1 / 2}+p^{2} \int_{0}^{T} \int_{M}\left(|\AA|^{2} \eta^{4}\right)^{2 p}\right) .
\end{aligned}
$$


Similarly we have the inequality

$$
\begin{aligned}
& p \int_{0}^{T} \int_{M}(H-h)^{2}\left(|\AA|^{2} \eta^{4}\right)^{2 p} \\
\leq & \left(\int_{0}^{T} \int_{\eta>0}(H-h)^{8}\right)^{1 / 4}\left(\left(\int_{0}^{T} \int_{M}\left(|\AA|^{2} \eta^{4}\right)^{4 p}\right)^{1 / 2}+p^{2} \int_{0}^{T} \int_{M}\left(|\AA|^{2} \eta^{4}\right)^{2 p}\right) .
\end{aligned}
$$

If we take a cutoff function $\eta$ with $\left.\eta\right|_{[0, T / 4]}=0$, then Lemma 24 implies

$$
\int_{0}^{T} \int_{\eta>0}(H-h)^{8}+\int_{0}^{T} \int_{\eta>0}|\AA|^{8} \leq C(\Lambda, T, v) E(T)^{2} .
$$

Hence, if $E(T)$ is sufficiently small, Lemma 23 together with (4-26) and (4-27) yields

$$
\left(\int_{0}^{T} \int_{M}\left(|\AA|^{2} \eta^{4}\right)^{4 p}\right)^{1 / 2} \leq c p^{2} \int_{0}^{T} \int_{M}\left(\Lambda^{2}+c_{\eta}\right)\left(|\AA|^{2} \eta^{4}\right)^{2 p}
$$

and

$$
\begin{array}{r}
\left(\int_{0}^{T} \int_{M}\left(|H-h|^{2} \eta^{4}\right)^{4 p}\right)^{1 / 2} \leq c p^{2} \int_{0}^{T} \int_{M}\left(\Lambda^{2}+\right. \\
\left.c_{\eta}+v^{-1}\right)\left(|H-h|^{2} \eta^{4}\right)^{2 p} \\
+c p v^{-1} \int_{0}^{T} \int_{M}(H-h)^{2},
\end{array}
$$

where we have used the fact that for any cutoff function $\eta$ with $\left.\eta\right|_{[0, T / 4]}=0$, the inequality (4-16) implies

$$
\sup _{t \in[0, T]}\left|h^{\prime} \eta^{4}\right| \leq \sup _{t \in[T / 4, T]}\left|h^{\prime}\right| \leq c / v_{0} .
$$

For each integer $k \geq 1$, let $b_{k}=(T / 2)\left(1-1 / 2^{k+1}\right)$ and $\eta_{k}=\eta_{0}\left(t-b_{k}\right) /\left(b_{k+1}-b_{k}\right)$, where $\eta_{0}$ is given by (4-18). Note that $\left.\eta_{k}\right|_{[0, T / 4]}=0$ for all $k \geq 1$. Hence, we can choose $c_{\eta_{k}}=2^{k+4} T^{-1}$. Taking $\eta=\eta_{k}$ and $p=2^{k-1}$ in (4-28), we have

$$
\left(\int_{b_{k+1}}^{T} \int_{M}\left(|\AA|^{2}\right)^{2^{k+1}}\right)^{2^{-(k+1)}} \leq\left(c 2^{2 k-2}\left(\Lambda^{2}+2^{k+4} T^{-1}\right)\right)^{2^{-k}}\left(\int_{b_{k}}^{T} \int_{M}\left(|\AA|^{2}\right)^{2^{k}}\right)^{2^{-k}} .
$$

Here we have used the fact that

$$
c_{\eta_{k}}\left(|\AA|^{2} \eta_{k}^{4}\right)^{2^{k}} \leq \frac{2^{k+4}}{T}\left(|\AA|^{2}\right)^{2^{k}} \eta_{k} .
$$

Iteration yields

$$
\sup _{M \times[T / 2, T]}|\AA|^{2} \leq c(\Lambda, T)\left(\int_{T / 4}^{T} \int_{M}|\AA|^{4}\right)^{1 / 2}
$$


Similarly, we can obtain the $L^{\infty}$ bound of $|H-h|$. In fact, we let

$$
I_{k}=\left(\int_{b_{k}}^{T} \int_{M}\left(|H-h|^{2}\right)^{2^{k}}\right)^{2^{-k}} .
$$

Then by assumption, (4-29) implies

$$
I_{k+1} \leq c_{k} \max \left\{I_{k}, \epsilon_{0}^{2^{-k}}\right\} \leq c_{k} \max \left\{I_{k}, 1\right\} \leq \max \left\{I_{1} \prod_{i=1}^{k} c_{i}, \sup _{1 \leq i \leq k} c_{i}\right\}
$$

where $c_{k}=\left(c\left(\Lambda^{2}+1 / v+2^{k+4} T^{-1}\right) 2^{2 k-2}\right)^{2^{-k}}$. It is easy to see that $\sup _{k} c_{k}<\infty$ and $\prod_{i=1}^{\infty} c_{i}<\infty$. Hence, taking $k \rightarrow \infty$ in the inequalities above, we have

$$
\sup _{t \in[T / 2, T]}|H-h|^{2} \leq C\left(\Lambda, T, v_{0}\right) .
$$

The proposition follows from this and (4-30).

4C. The proof of Proposition 18. Suppose $M_{0}$ satisfies the assumption (4-1). Let $\lambda>0$ be a parameter and define

$$
\begin{aligned}
t_{0}:=\sup \{0 \leq t \leq \min \{T, \lambda\}: \gamma / 10<h(\tau)< & 10 \Lambda, \int_{M}|H-h|^{2}(\tau)<10 \epsilon, \\
& \left.\int_{M}|\AA|^{2}(\tau) \leq 10 \epsilon \text { for all } \tau \in[0, t)\right\},
\end{aligned}
$$

where $T$ is the maximal time of smooth existence. By the definition of $t_{0}$, we have the inequalities

$$
\begin{aligned}
\gamma / 10<h(t) & <10 \Lambda, \\
\int_{M}|H-h|^{2}(t) & <10 \epsilon, \quad \int_{M}|\AA|^{2}(t) \leq 10 \epsilon \quad \text { for } t \in\left[0, t_{0}\right) .
\end{aligned}
$$

Now by Lemma 19, if we choose $10 \epsilon<\epsilon_{0}$, the assumption (4-1) implies

$$
\int_{M}|\AA|^{2} \leq \epsilon \quad \text { and } \quad \int_{0}^{t} \int_{M}|\AA|^{4} \leq 2 \epsilon \quad \text { for } t \in\left[0, t_{0}\right) .
$$

By Lemma 20 we have

$$
\int_{M}(H-h)^{2}(t)+\int_{0}^{t} \int_{M}(H-h)^{4} \leq 2 \epsilon e^{600 \Lambda^{2} \lambda}<3 \epsilon \quad \text { for } t \in\left[0, t_{0}\right],
$$

where we choose $\lambda<\lambda_{1}(\Lambda)<1$ sufficiently small. Hence we have

(4-33) $E(t)=\int_{0}^{t} \int_{M}(H-h)^{2}+|\AA|^{4}+(H-h)^{4} \leq 3 \epsilon \lambda+5 \epsilon<8 \epsilon \quad$ for $t \in\left[0, t_{0}\right)$. 
Now Lemma 21 implies

$$
h(t) \leq \Lambda+\frac{8 \epsilon}{V_{0}-8 \epsilon}<2 \Lambda
$$

and

$$
h(t) \geq \gamma-\frac{8 \epsilon}{V_{0}-8 \epsilon}>\frac{\gamma}{2}, \quad V_{t} \geq v_{0}-8 \epsilon \geq \frac{v_{0}}{2} \quad \text { for } t \in\left[0, t_{0}\right),
$$

where we choose $\lambda<\lambda_{2}\left(\Lambda, \gamma, v_{0}\right)<\lambda_{1}$ small. Now if $t_{0}=\lambda$, then (4-2) holds and the proposition is proved. Otherwise $t_{0}=T<\lambda$, which contradicts the fact that under the estimates (4-31)-(4-34), Proposition 22 implies the $L^{\infty}$ that the second fundamental form is bounded; that is, $|A|(T) \leq C$, and the solution can be extended beyond $T$.

\section{Acknowledgments}

I would like to thank Professor F. Pacard for support and encouragement during the course of this work. I also thank Professor X. X. Chen, W. Y. Ding and X. H. Zhu for their help over the past several years.

\section{References}

[Alikakos and Freire 2003] N. D. Alikakos and A. Freire, "The normalized mean curvature flow for a small bubble in a Riemannian manifold", J. Differential Geom. 64:2 (2003), 247-303. MR 2004i: 53094 Zbl 1070.53038

[Cabezas-Rivas and Miquel 2007] E. Cabezas-Rivas and V. Miquel, "Volume preserving mean curvature flow in the hyperbolic space", Indiana Univ. Math. J. 56:5 (2007), 2061-2086. MR 2008i: 53089 Zbl 1130.53045

[Chen 2006] X. Chen, "On the lower bound of energy functional $E_{1}$, I: A stability theorem on the Kähler Ricci flow”, J. Geom. Anal. 16:1 (2006), 23-38. MR 2006j:53096

[Chen and Li 2009] X. X. Chen and H. Li, "Stability of Kähler-Ricci flow", preprint, 2009. To appear in J. of Geom. Anal.

[Chen and Tian 2006] X. X. Chen and G. Tian, "Ricci flow on Kähler-Einstein manifolds", Duke Math. J. 131:1 (2006), 17-73. MR 2007c:53083

[Chen et al. 2009] X. Chen, H. Li, and B. Wang, "Kähler-Ricci flow with small initial energy", Geom. Funct. Anal. 18:5 (2009), 1525-1563. MR 2481736

[Dai et al. 1996] X. Dai, G. Wei, and R. Ye, "Smoothing Riemannian metrics with Ricci curvature bounds”, Manuscripta Math. 90:1 (1996), 49-61. MR 97b:53039 Zbl 0880.53034

[Ecker 1995] K. Ecker, "On regularity for mean curvature flow of hypersurfaces", Calc. Var. Partial Differential Equations 3:1 (1995), 107-126. MR 97f:58040 Zbl 0814.53004

[Escher and Simonett 1998] J. Escher and G. Simonett, "The volume preserving mean curvature flow near spheres”, Proc. Amer. Math. Soc. 126:9 (1998), 2789-2796. MR 98m:53054 Zbl 0909.53043

[Gage and Hamilton 1986] M. Gage and R. S. Hamilton, "The heat equation shrinking convex plane curves", J. Differential Geom. 23:1 (1986), 69-96. MR 87m:53003 Zbl 0621.53001

[Huisken 1984] G. Huisken, "Flow by mean curvature of convex surfaces into spheres", J. Differential Geom. 20:1 (1984), 237-266. MR 86j:53097 Zbl 0556.53001 
[Huisken 1987] G. Huisken, “The volume preserving mean curvature flow”, J. Reine Angew. Math. 382 (1987), 35-48. MR 89d:53015 Zbl 0621.53007

[Huisken and Yau 1996] G. Huisken and S.-T. Yau, "Definition of center of mass for isolated physical systems and unique foliations by stable spheres with constant mean curvature", Invent. Math. 124:1-3 (1996), 281-311. MR 96m:53037 Zbl 0858.53071

[Kuwert and Schätzle 2002] E. Kuwert and R. Schätzle, "Gradient flow for the Willmore functional", Comm. Anal. Geom. 10:2 (2002), 307-339. MR 2003c:53099 Zbl 1029.53082

[Mazzeo and Pacard 2007] R. Mazzeo and F. Pacard, "Constant curvature foliations in asymptotically hyperbolic spaces”, preprint, 2007. arXiv 0710.2298v2

[Michael and Simon 1973] J. H. Michael and L. M. Simon, "Sobolev and mean-value inequalities on generalized submanifolds of $\mathbb{R}^{n}$ ", Comm. Pure Appl. Math. 26 (1973), 361-379. MR 49 \#9717

[Min-Oo 1990] M. Min-Oo, "Almost Einstein manifolds of negative Ricci curvature", J. Differential Geom. 32:2 (1990), 457-472. MR 91g:53047 Zbl 0725.53050

[Nakauchi 1993] N. Nakauchi, "A concentration phenomenon in mean curvature flow", Manuscripta Math. 78:2 (1993), 129-147. MR 93k:58060 Zbl 0795.53056

[Rigger 2004] R. Rigger, "The foliation of asymptotically hyperbolic manifolds by surfaces of constant mean curvature (including the evolution equations and estimates)", Manuscripta Math. 113:4 (2004), 403-421. MR 2005k:53109 Zbl 1065.53029

[Topping 2008] P. Topping, "Relating diameter and mean curvature for submanifolds of Euclidean space”, Comment. Math. Helv. 83:3 (2008), 539-546. MR 2009b:53100 Zbl 1154.53007

[Ye 1993] R. Ye, "Ricci flow, Einstein metrics and space forms", Trans. Amer. Math. Soc. 338:2 (1993), 871-896. MR 93j:58029 Zbl 0804.53054

Received July 1, 2008. Revised May 25, 2009.

\section{HAOZHAO LI}

DEPARTMENT OF MATHEMATICS

EAST CHINA NORMAL UNIVERSITY

SHANGHAI 200241

CHINA

lihaozhao@gmail.com 